\title{
FINITELY GENERATED SOLUBLE GROUPS WITH A CONDITION ON INFINITE SUBSETS
}

\author{
ASADOLLAH FARAMARZI SALLES
}

(Received 2 March 2012; accepted 8 May 2012)

\begin{abstract}
Let $G$ be a group. We say that $G \in \mathcal{T}(\infty)$ provided that every infinite set of elements of $G$ contains three distinct elements $x, y, z$ such that $x \neq y,[x, y, z]=1=[y, z, x]=[z, x, y]$. We use this to show that for a finitely generated soluble group $G, G / Z_{2}(G)$ is finite if and only if $G \in \mathcal{T}(\infty)$.
\end{abstract}

2010 Mathematics subject classification: primary 20F19.

Keywords and phrases: finitely generated groups, nilpotent groups, soluble groups.

\section{Introduction}

Paul Erdős [10] posed the following question. Suppose that every infinite set of elements of a group $G$ contains a pair of elements which commute. Does there exist an upper bound for the order of (finite) subsets of $G$ consisting of pairwise noncommuting elements?

An affirmative answer to this question was given by Neumann [10] who proved that an infinite group $G$ is centre-by-finite if and only if every infinite subset of $G$ contains two distinct commuting elements. Since this paper, problems of a similar nature have been the object of several articles (for example, [1-10]).

Let $G$ be a group and $\chi$ a class of groups. We say that $G$ satisfies the condition $(\chi, \infty)$ if every infinite subset of $G$ contains a pair of elements which generate a subgroup in the class $\chi$. We also say that $G$ satisfies condition $\mathcal{T}(\infty)$ (or $G$ is in $\mathcal{T}(\infty)$ ) if every infinite set of elements of $G$ contains three elements $x, y, z$ such that

$$
x \neq y, \quad[x, y, z]=1=[y, z, x]=[z, x, y] .
$$

Our terminology and notation are standard and follow [4]. In this paper $Z_{n}(G)$ denotes the $(n+1)$ th term of the upper central series of $G$, and $\Gamma_{n}(G)$ denotes the $n$th term of the lower central series of $G$. Let $\mathcal{N}_{2}$ and $\mathcal{E}_{2}$ be the classes of nilpotent groups of class at most 2 and 2-Engel, respectively. Obviously

$$
\left(\mathcal{N}_{2}, \infty\right) \subseteq\left(\mathcal{E}_{2}, \infty\right) \subseteq \mathcal{T}(\infty)
$$

(C) 2012 Australian Mathematical Publishing Association Inc. 0004-9727/2012 \$16.00 
In [3], Delizia proved that a finitely generated soluble group $G$ is in $\left(\mathcal{N}_{2}, \infty\right)$ if and only if $G / Z_{2}(G)$ is finite. In [2], Abdollahi proved that a finitely generated soluble group $G$ is in $\left(\mathcal{E}_{2}, \infty\right)$ if and only if $G / Z_{2}(G)$ is finite.

In this paper, we prove the following theorem.

Main Theorem. Let $G$ be a finitely generated soluble group. Then $G \in \mathcal{T}(\infty)$ if and only if $G / Z_{2}(G)$ is finite.

The main theorem implies that, for a finitely generated soluble group $G$, the following conditions are equivalent:

$$
G \in\left(\mathcal{N}_{2}, \infty\right), \quad G \in\left(\mathcal{E}_{2}, \infty\right), \quad G \in \mathcal{T}(\infty), \quad G / Z_{2}(G) \text { is finite }
$$

\section{Results}

In the first result we prove the sufficiency.

Lemma 2.1. Let $G$ be a group and suppose that $G / Z_{2}(G)$ is finite. Then $G \in \mathcal{T}(\infty)$.

Proof. Let $X$ be an infinite subset of $G$. There exists an infinite subset $X_{0}$ of $X$ such that $x Z_{2}(G)=y Z_{2}(G)=z Z_{2}(G)$, for $x, y, z \in X_{0}$. So $[x, y, z]=[y, z, x]=[z, x, y]=1$.

Recall that a group $G$ is called a restrained group if $\langle x\rangle^{\langle y\rangle}$ is finitely generated for all $x, y \in G$.

Proposition 2.2. Every group in $\mathcal{T}(\infty)$ is a restrained group.

Proof. Let $G$ be a group in $\mathcal{T}(\infty)$ and $x, y \in G$ such that $y$ has infinite order. Since $X=\left\{x y^{i} \mid i>1\right\}$ is an infinite subset of $G$, there exist three integers $i<j \leq k$ such that

$$
\left[x y^{i}, x y^{j}, x y^{k}\right]=\left[x y^{j}, x y^{k}, x y^{i}\right]=\left[x y^{k}, x y^{i}, x y^{j}\right]=1 .
$$

It follows from the equations $\left[x y^{t}, x y^{s}\right]=x^{-y^{t}} x^{y^{s}}$ and (2.1) that

$$
\begin{aligned}
& x y^{k-j} x^{-1} y^{j-i} x y^{i-k} x x^{-y^{i}} x^{y^{j}}=1, \\
& x y^{i-k} x^{-1} y^{k-j} x y^{j-i} x x^{-y^{j}} x^{y^{k}}=1, \\
& x y^{j-i} x^{-1} y^{i-k} x y^{k-j} x x^{-y^{k}} x^{y^{i}}=1,
\end{aligned}
$$

and so $x^{y^{k}}=x^{y^{i}} x^{-1} x^{-y^{j-i}} x^{y^{k-i}} x^{-1}$. In this case we conclude that

$$
\left\langle x^{y^{i}}: i \geq 0\right\rangle \leq\left\langle x^{y^{n}}:|n|<k\right\rangle .
$$

Now starting from the infinite set $X=\left\{x y^{i} \mid i<1\right\}$ and repeating the previous argument, we can prove that

$$
\left\langle x^{y^{i}}: i \leq 0\right\rangle \leq\left\langle x^{y^{n}}:|n|<k^{\prime}\right\rangle,
$$

for a suitable integer $k^{\prime}>1$. Therefore there exists a positive integer $m$ such that $\langle x\rangle^{\langle y\rangle}=\left\langle x^{y^{n}}:|n|<m\right\rangle$. 
LeMma 2.3. Let $G$ be a finitely generated group in $\mathcal{T}(\infty)$. If $G / Z_{3}(G)$ is finite, then so is $Z_{2}\left(Z_{3}(G)\right) / Z_{2}(G)$.

Proof. It is clear that $Z_{2}(G) \leq Z_{2}\left(Z_{3}(G)\right)$. Let $x \in Z_{2}\left(Z_{3}(G)\right) \leq Z_{3}(G)$. Then, for any $y, z, t \in G$,

$$
[x, y, z, t]=1, \quad[x, y, z]^{t}=\left[x, y, z^{t}\right]=[x, y, z] .
$$

Let $\left|G / Z_{3}(G)\right|=n$. It follows that, for any $y, z \in G,\left[x, y^{n}, z^{n}\right]=1$ and so, by (2.2), $[x, y, z]^{n^{2}}=1=\left[x^{n^{2}}, y, z\right]$. Thus $x^{n^{2}} \in Z_{2}(G)$ and $Z_{2}\left(Z_{3}(G)\right) / Z_{2}(G)$ has finite exponent dividing $n^{2}$. Now $Z_{2}\left(Z_{3}(G)\right) / Z_{2}(G)$ is a finitely generated nilpotent torsion group and thus finite as required.

Lemma 2.4. Let $G$ be a finitely generated nilpotent group of class at most 3 which satisfies $\mathcal{T}(\infty)$. Then $G / Z_{2}(G)$ is finite.

Proof. We consider the following cases.

Case I. Let $G$ be a torsion group. Then $G$ is a finitely generated nilpotent torsion group and thus finite.

Case II. Let $G$ be a torsion-free group. We claim that $G=Z_{2}(G)$. Since $G$ is nilpotent of class at most 3 ,

$$
\left[x^{n}, y^{m}, z^{k}\right]=[x, y, z]^{n m k}, \quad[x, y, z]^{g}=[x, y, z],
$$

for all $g, x, y, z$ in $G$ and all integers $m, n, k$. Now consider the infinite subset $X=\left\{x y^{1}, x y^{2}, x y^{3}, \ldots\right\}$ of $G$. Since $G$ is in $\mathcal{T}(\infty)$, there exist three positive integers $i \neq j, k$ such that

$$
\left[x y^{i}, x y^{j}, x y^{k}\right]=1=\left[x y^{j}, x y^{k}, x y^{i}\right]=\left[x y^{k}, x y^{i}, x y^{j}\right] .
$$

Repeated application of (2.3) yields

$$
1=\left[x y^{i}, x y^{j}, x y^{k}\right]=\left([y, x, x][x, y, y]^{-k}\right)^{i-j} .
$$

Since $G$ is torsion-free, $[x, y, y]^{k}=[y, x, x]$ and also $[x, y, y]^{j}=[y, x, x]$. Therefore $[x, y, y]^{k-j}=1$, and hence $[x, y, y]=1$. Thus $G$ is a 2-Engel group. Now since $G$ is metabelian [11, Theorem 7.36] implies that $\Gamma_{3}(G)=1$, and so $G=Z_{2}(G)$.

Case III. Let $G$ be neither a torsion nor a torsion-free group. Then $G / G_{t}$ is a torsionfree group, where $G_{t}$ is a torsion subgroup of the nilpotent group $G$. Since $\mathcal{T}(\infty)$ is closed under taking subgroups and homomorphic images, we have by Case II that $G / G_{t}$ is nilpotent of class 2 and thus $\Gamma_{3}(G) \leq G_{t}$ is finite. Therefore $G / Z_{2}(G)$ is finite.

Proposition 2.5. Let $G$ be a finitely generated nilpotent group of class $c$ in $\mathcal{T}(\infty)$. Then $G / Z_{2}(G)$ is finite. 
Proof. We argue by induction on $c$. Since $G / Z(G)$ is nilpotent of class $c-1$, we have that $G / Z_{3}(G)$ is finite. Now $Z_{3}(G) / Z_{2}\left(Z_{3}(G)\right)$ is also finite by Lemma 2.4. The result follows from Lemma 2.3.

The following result is analogous to [4, Lemma 1].

Lemma 2.6. Let $G$ be an infinite residually finite group satisfying the condition $\mathcal{T}(\infty)$. Then the centraliser $C_{G}(x)$ is infinite, for all $x$ in $G$.

Proof. Suppose, for a contradiction, that $G$ has an element $x$ with finite centraliser $C_{G}(x)$. Since $G$ is residually finite, there exists a normal subgroup $N$ of $G$ such that $N \cap C_{G}(x)=1$ and $G / N$ is finite. In particular, $N$ is infinite. Consider the infinite set $\left\{x^{n}: n \in N\right\}$. Then, by the property $\mathcal{T}(\infty)$, there exist three elements $r, s, t \in N$ such that $r \neq s,\left[x^{r}, x^{s}, x^{t}\right]=\left[x^{t}, x^{r}, x^{s}\right]=\left[x^{s}, x^{t}, x^{r}\right]=1$. Now the equation $\left[x^{r}, x^{s}, x^{t}\right]=1$ implies that $\left[x^{p}, x^{q}, x\right]=1$ with $p=r t^{-1} \in N$ and $q=s t^{-1} \in N$. It follows that $\left[x^{p}, x^{q}\right] \in$ $N \cap C_{G}(x)=1$, since $\left[x^{p q^{-1}}, x\right]=\left[q p^{-1}, x\right]^{x p q^{-1}}\left[p q^{-1}, x\right] \in N$. Hence $\left[x^{p}, x^{q}\right]=1$ and $x^{p q^{-1}} \in C_{G}(x)$. Since $x^{p q^{-1}}=\left[p q^{-1}, x^{-1}\right] x$, we get $\left[p q^{-1}, x^{-1}\right] \in N \cap C_{G}(x)=1$ and $p q^{-1} \in N \cap C_{G}(x)=1$, so $p=q$. We thus obtain the contradiction that $r=s$.

COROLlary 2.7. Let $G$ be an infinite residually finite group satisfying the condition $\mathcal{T}(\infty)$. Then every element $x$ of $G$ is contained in an infinite abelian subgroup of $G$.

Lemma 2.8. Let $G$ be an infinite residually finite group satisfying the condition $\mathcal{T}(\infty)$. Then the centraliser $C_{G}(X)$ is infinite, for any finite subset $X$ of $G$.

Proof. The proof is by induction on $m=|X|$. If $m=1$, the result is true by Lemma 2.6. Suppose that $m>1, X=\left\{x_{1}, \ldots, x_{m}\right\}$ and $C_{G}\left(x_{1}, \ldots, x_{m-1}\right)$ is infinite. Then, by Corollary 2.7, there exists an infinite abelian subgroup $A$ of $G$ such that $A \leq C_{G}\left(x_{1}, \ldots, x_{m-1}\right)$. Put $x_{m}=x$. Since $G$ is residually finite, there exists an infinite descending sequence $\left(N_{i}\right)_{i \in I}$ of normal subgroups of $G$ with $G / N_{i}$ finite for any $i \in I$ and $\cap N_{i}=1$. Therefore, $A \cap N_{i}$ is infinite for any $i \in I$.

Now, as in the proof of [4, Lemma 3], we can prove that there exist a sequence $\left(a_{n}\right)_{n \in \mathbb{N}}$ of elements of $A$ that are pairwise distinct and a subsequence $\left(M_{n}\right)_{n \in \mathbb{N}}$ of $\left(N_{i}\right)_{i \in I}$ such that for every $n \in \mathbb{N}$ we get $a_{n+1} \in M_{n}$ and either $\left[a_{n}, x, x\right]=1$ or $\left[a_{n}, x, x\right] \notin M_{n}$. Moreover, if $\left[a_{n}, x, x\right]=1$ and $\left[a_{n}, x, x_{s}\right] \neq 1$ for some $s \in\{1, \ldots$, $m-1\}$ then $\left[a_{n}, x, x_{s}\right]^{x} \notin M_{n}$. Now we consider the infinite set $\left\{a_{1} x, \ldots, a_{n} x, \ldots\right\}$. Since $G$ satisfies the condition $\mathcal{T}(\infty)$, there exist $i, j, k \in \mathbb{N}$ with $i<j \leq k$ such that $\left[a_{i} x, a_{j} x, a_{k} x\right]=1$ and $\left[a_{i} x, a_{j} x, x\right]\left[a_{i} x, a_{j} x, a_{k}\right]^{x}=1$; then $\left[a_{i} x, a_{j} x, x\right] \in\left\langle a_{k}\right\rangle^{G} \leq$ $M_{k-1} \leq M_{i}$. Since $\left[a_{i} x, a_{j}, x\right] \in\left\langle a_{j}\right\rangle^{G} \leq M_{j-1} \leq M_{i}$, we have $\left[a_{i} x, x, x\right] \in M_{i}$ and then $\left[a_{i}, x, x\right] \in M_{i}$ which implies that $\left[a_{i}, x, x\right]=1$. So $B=\left\{a \in\left(a_{n}\right)_{n \in \mathbb{N}}:[a, x, x]=1\right\}$ is an infinite set. Suppose that $B=\left\{b_{1}, \ldots, b_{n}, \ldots\right\}$. Let $x_{l} \in\left\{x_{1}, \ldots, x_{m-1}\right\}$, and consider the infinite set $\left\{b_{i} x_{l} x: b_{i} \in B\right\}$. Then there exist $r, s, t \in \mathbb{N}$ with $r<$ $s \leq t$ such that $\left[b_{r} x_{l} x, b_{s} x_{l} x, b_{t} x_{l} x\right]=1$. Since $\left[b_{r} x_{l} x, b_{s} x_{l} x, b_{t}\right] \in\left\langle b_{t}\right\rangle^{G} \leq M_{t-1} \leq M_{r}$, we have $\left[b_{r} x_{l} x, b_{s} x_{l} x, x_{l} x\right] \in M_{s} \leq M_{r}$. Since $\left[b_{r} x_{l} x, b_{s}, x_{l} x\right] \in\left\langle b_{s}\right\rangle^{G} \leq M_{s-1} \leq M_{r}$, we have $\left[b_{r}, x_{l} x, x_{l} x\right] \in M_{r}$. It follows that $\left[b_{r}, x, x_{l}\right] \in M_{r}$ and then $\left[b_{r}, x, x_{l}\right]=1$, as $b_{r} \in C_{G}\left(x_{1}, \ldots, x_{m-1}\right)$. Thus there exists an infinite subset $B^{*}$ of $B$ such that 
$\left[b, x, x_{l}\right]=1$ for any $b \in B^{*}$. We can now easily prove that there exists an infinite subset $V$ of $B$ such that $[c, x] \in C_{G}\left(x_{1}, \ldots, x_{m-1}\right)$ for every $c \in V$. If the set $\{[c, x]: c \in V\}$ is infinite the result follows. Otherwise, there exist $c \in B$ and an infinite subset $\left\{d_{j}: j \in J\right\} \subseteq B$ such that $[c, x]=\left[d_{j}, x\right]$ for any $j \in J$. Then the infinite set $\left\{c d_{j}^{-1}: j \in J\right\}$ is contained in $C_{G}\left(x_{1}, \ldots, x_{m-1}\right)$, and the result follows.

The following is an immediate corollary of Lemma 2.8.

CoRollary 2.9. Let $G$ be a finitely generated infinite residually finite group in $\mathcal{T}(\infty)$. Then $Z(G)$ is infinite.

Denote by $h l(G)$ the Hirsch length of $G$.

Lemma 2.10. Let $G$ be a finitely generated infinite polycyclic group in $\mathcal{T}(\infty)$. Then $G / Z_{2}(G)$ is finite.

Proof. If $h l(G)=1$ then, by Corollary 2.9, $G / Z(G)$ is finite. Suppose then that $h l(G)>1$. It follows that $h l(G)>h l(G / Z(G))$. Now, by the induction hypothesis,

$$
\frac{G / Z(G)}{Z_{2}(G / Z(G))} \cong \frac{G}{Z_{3}(G)}
$$

is finite. Therefore, the result follows from Lemma 2.3 and Proposition 2.5.

Proof OF THE MAIN THEOREM. To show that a finitely generated soluble group $G$ in $\mathcal{T}(\infty)$ has $G / Z_{2}(G)$ finite, it is enough to show that $G$ is polycyclic by Lemma 2.10. It follows from Proposition 2.2 that $G^{\prime}$ is finitely generated. Since a finitely generated abelian group is polycyclic and the class of a polycyclic group is closed under extensions, induction on the derived length then gives us $G$ polycyclic. The other direction follows immediately from Lemma 2.1.

Corollary 2.11. Let $G$ be a finitely generated soluble group. Then the following conditions are equivalent.

(i) $G \in\left(\mathcal{N}_{2}, \infty\right)$.

(ii) $G \in\left(\mathcal{E}_{2}, \infty\right)$.

(iii) $G \in \mathcal{T}(\infty)$.

(iv) $G / Z_{2}(G)$.

Proof. This follows using also the main theorems of $[2,3]$.

\section{Acknowledgement}

The author would like to thank Professor G. Traustason and the referee for useful suggestions. 


\section{References}

[1] A. Abdollahi, 'Some Engel conditions on infinite subsets of certain groups', Bull. Aust. Math. Soc. 62 (2000), 141-148.

[2] A. Abdollahi, 'Finitely generated soluble groups with an Engel condition on infinite subsets', Rend. Semin. Mat. Univ. Padova 103 (2000), 47-49.

[3] C. Delizia, 'Finitely generated soluble groups with a condition on infinite subsets', Ist. Lombardo Accad. Sci. Lett. Rend. A 128 (1994), 201-208.

[4] C. Delizia, 'On certain residually finite groups', Comm. Algebra 24 (1996), 3531-3535.

[5] G. Endimioni, 'Groups covered by finitely many nilpotent subgroups', Bull. Aust. Math. Soc. 50 (1994), 459-464.

[6] G. Endimioni, 'Groupes finis satisfaisant la condition $(\mathcal{N}, n)$ ', C. R. Acad. Sci. Paris Sér. I Math. 319 (1994), 1245-1247.

[7] J. C. Lennox and J. Wiegold, 'Extensions of a problem of Paul Erdôs on groups', J. Aust. Math. Soc. Ser. A 31 (1981), 459-463.

[8] P. Longobardi, 'On locally graded groups with an Engel condition on infinite subsets', Arch. Math. (Basel) 76 (2001), 88-90.

[9] P. Longobardi and M. Maj, 'Finitely generated soluble groups with an Engel condition on infinite subsets', Rend. Semin. Mat. Univ. Padova 89 (1993), 97-102.

[10] B. H. Neumann, 'A problem of Paul Erdős on groups', J. Aust. Math. Soc. Ser. A 21 (1976), 467-472.

[11] D. J. Robinson, Finiteness Condition and Generalized Soluble Groups, Part 1, 2 (Springer, Berlin, 1972).

ASADOLLAH FARAMARZI SALLES, Department of Mathematics, Damghan University, Damghan, Iran

e-mail: faramarzi@du.ac.ir 